\title{
Home parenteral nutrition (HPN): a Welsh experience
}

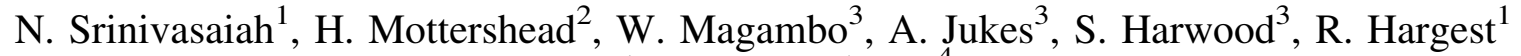 \\ and A. B. Hawthorne \\ ${ }^{1}$ Department of Surgery, Cardiff University, Cardiff, UK, ${ }^{2}$ Cardiff University, Cardiff, UK, ${ }^{3}$ University Hospital of Wales \\ Nutrition Team, Cardiff CF14 4XW, UK and ${ }^{4}$ Department of Gastroenterology, University Hospital of Wales, \\ Cardiff CF14 4 XW, UK
}

Since establishment of HPN training in Wales, there has been a rapid increase in numbers on long-term HPN, from $7.3 / 10^{6}$ in 2002 , to $20 / 10^{6}$ currently. HPN training has been undertaken at the University Hospital of Wales (UHW) since 2000. We review here the outcomes and complications of home parenteral nutrition (HPN) in Cardiff since establishment of the service.

Fifty patients have been managed at home on HPN since 2000. Of these 43 were trained at UHW (7 were trained in Manchester or St Marks prior to 2000). We present a review of outcomes of therapy, particularly mortality and duration of feeding. For patients currently on feeding, catheter-related complications (infection and thrombosis), level of bone disease and liver function are presented. Data were collected from the HPN database and electronic results reporting and clinical notes. Infection rates are presented for the year April 2008March 2009 and presented as catheter-related infections (CRI) per 1000 feed days during this time. SPSS was used for statistical analysis.

Crohn's and infarction were two major aetiological factors for intestinal failure accounting for 17 (34\%) and 9 (18\%), respectively. Fistulae $7(14 \%)$, radiation enteritis $2(14 \%)$ and enteropathy $8(16 \%)$ (SLE, Scleroderma, Chronic Pancreatitis, Intestinal lymphangiectasia, MCTD) were some of the other causes. Of the 50 patients, 8 have died (only 1 death was HPN-related (haemorrhage as a consequence of anticoagulation following multiple large vein thromboses)). All other deaths were due to underlying disease. HPN was stopped after planned restorative surgery in 7 patients, and 2 were able to manage on subcutaneous fluids and magnesium without HPN. The remaining 33 are still on HPN. Nineteen (57.6\%) female, with a median age of 57 years (range 17-77). No patients have been accepted onto a small intestinal transplant waiting list.

Complications in patients currently on HPN $(n=33)$

Infection: Over the year 2008-9, 6 (20\%) had documented CRI. Four occurred in out-patients giving a rate of 0.43 CRI/1000 feed days, with three treated with antibiotics and catheter salvage and only one requiring line replacement. Two infections occurred prior to initial discharge from hospital. Two significant infective complications occurred (cervical discitis treated with long-term antibiotics and cervical osteomyelitis treated with surgery and antibiotics). The latter infection was likely related to previous intravenous pethidine use for chronic pain.

Metabolic bone disease: In spite of widespread use of intravenous bisphosphonates, there is significant bone loss, correlating with duration of HPN $(r=0.403, P=0.022)$. Five patients have severe symptomatic osteoporosis with kyphosis and/or fractures. In the total cohort, the average "T" score was -1.8 (SD 1.6) for lumbar spine and -2.0 (SD 1.2) for Hip. The lumbar spine was normal, osteopenic and osteoporotic in $7(21 \%), 13(39 \%), 8(24 \%)$ patients, respectively. For the Hip, it was normal, osteopenic and osteoporotic in $4(12 \%), 17(51 \%)$ and $7(21 \%)$ patients, respectively. Twenty five OH vitamin D was $25 \mu \mathrm{g} / \mathrm{l}$ (SD 14), but many patients were on replacement with various vitamin D preparations. As a better marker of ionised calcium and vitamin D status, average serum PTH level was $7.7 \mathrm{pmol} / \mathrm{l}$ (normal range 0.9-5.4) and 16 (53.3\%) patients had high PTH levels.

Liver function: Alkaline phosphatase and GGT were raised (>x2 upper limit of normal) in 8 (24\%) and 18 (54\%), respectively. Aspartate transaminase was high ( > x2 upper limit of normal) only in $2(6 \%)$ patients. There was hyperbilirubinaemia with jaundice in a minority $3(9 \%)$ who were recently started on HPN. Eighteen $(54.5 \%)$ have had liver imaging with 8 (44\%) showed abnormalities (fatty liver or hepatomegaly, and one has a clinical diagnosis of cirrhosis). No patient has undergone liver biopsy.

We have had a rapid increase in Welsh patients on HPN, and our unit has demonstrated low levels of serious complications, with only one death related directly to HPN. Metabolic bone disease and liver functions are common sequelae of HPN, and require regular monitoring within a specialised service because of the potential for significant future morbidity. 